\title{
COVID-19 and stroke: a case report
}

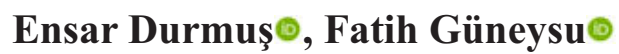 \\ Department of Emergency Medicine, Sakarya University Training and Research Hospital, Sakarya, Turkey
}

\begin{abstract}
COVID-19 disease is a worldwide pandemic. Patients present to hospitals with upper and lower respiratory tract symptoms such as cough, fever, shortness of breath, however it can also be encountered with many different clinics. We aimed to share the patient who has COVID-19 and presented with stroke. A 67-year-old female patient presented to the emergency service complaints of mental fog and shortness of breath. She had known lung cancer, hypertension and diabetes. There were no signs compatible with COVID-19 in her history or examination. No feature was detected in her blood tests. Diffuse cerebral infarction was observed in diffusion MRI examination. The patient, whose general condition was poor, was taken into follow up in intensive care unit. On the eighth day of her follow-up, the patient passed away. In places where COVID-19 disease is observed commonly, it would be appropriate for emergency physicians to take COVID-19 disease into consideration in stroke cases.

Keywords: COVID-19, stroke, pandemic
\end{abstract}

\begin{abstract}
COVID-19 disease is a worldwide pandemic. While most cases overcome the disease with mild symptoms, disease progresses much more severe in some cases. Patients present to hospitals with upper and lower respiratory tract symptoms such as cough, fever, shortness of breath, however it can also be encountered with many different clinics. We aimed to share the patient who has COVID-19 and presented to our emergency department with stroke.
\end{abstract}

\section{CASE PRESENTATION}

A 67-year-old female patient was tried to be woken up for breakfast in the morning of 23/03/2020 by her son, however she was brought to our emergency service by ambulance when it was noticed that she had mental fog and limited motion. She had known history of lung cancer, hypertension, and diabetes. She last had chemical treatment on 18/03/2020. On $23 / 03 / 2020$, she presented to the emergency service of Sakarya University Training and Research Hospital with complaints of mental fog and shortness of breath. Blood pressure was 160/90 $\mathrm{mmHg}$, pulse was 82 beats per min, respiratory rate was 26 , fever was $36.8^{\circ} \mathrm{C}$, oxygen saturation was 92 in room air and 98 with oxygen, and blood sugar was $82 \mathrm{mg} / \mathrm{dL}$. Her electrocardiogram showed normal sinus rhythm. Her general condition was poor, her consciousness had tendency for sleep, there was no co-operation, and she responded to painful stimuli with a grimace. Pupillary was isochoric, light reflex was bilateral $+/+$, four extremities were partially mobile. According to the information received from her family, until the evening of 22/03/2020, she ran her errands, she could eat on her own, go to the toilet on her own, had normal 


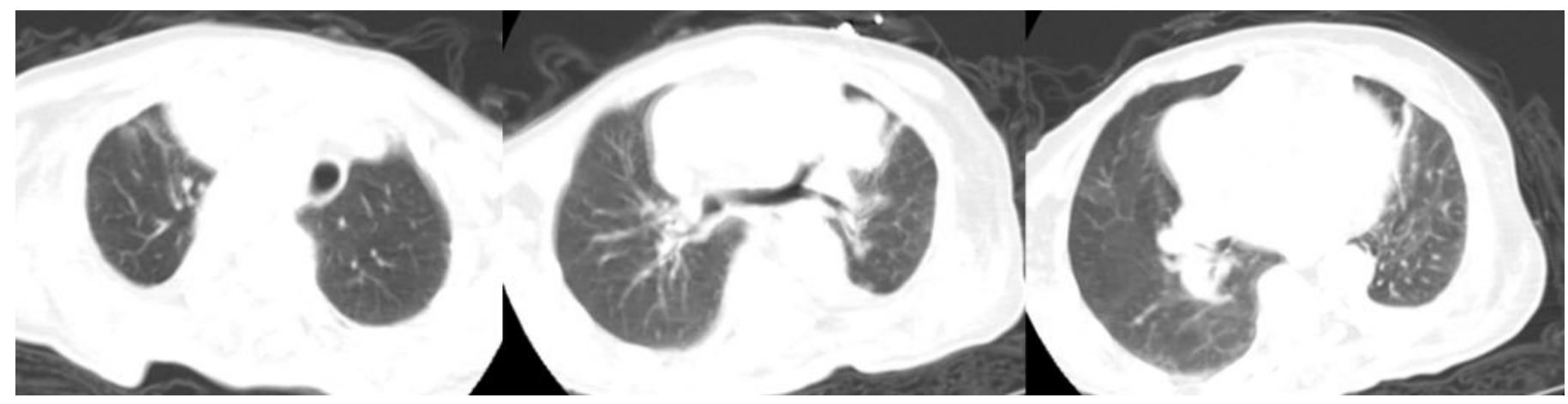

Fig. 1. Thorax computed tomographic imaging

speech and she had a history of suspected cranial metastasis.

Hemogram results showed hemoglobin as $10.8 \mathrm{~g} / \mathrm{dL}$, platelet as $140 \mathrm{~K} / \mu \mathrm{L}$ and lymphocyte as $1.81 \mathrm{~K} / \mu \mathrm{L}$. Among the biochemistry parameters, CK-MB was measured as $27.5 \mathrm{U} / \mathrm{L}$, urea as $46 \mathrm{mg} / \mathrm{dL}$, and high sensitive Troponin I (hsTnI) as $429.1 \mathrm{ng} / \mathrm{L}$. In blood gases, lactate was found as $0.6 \mathrm{mmol} / \mathrm{L}$, $\mathrm{pH}$ as 7.349 and $\mathrm{pCO}_{2}$ as $33.8 \mathrm{mmHg}$.

Brain and thorax non-contrasted computed tomography was performed to detect central pathologies especially and since she had shortness of breath. Brain tomography revealed low-density hyperdense areas in the left frontoparietal lobe, and cortical and subcortical hypodense areas in the right occipital lobe. The patient was consulted in the neurology department urgently and it was decided to perform cranial diffusion MRI to better distinguish between infarction and brain metastasis. Thorax tomography images of 23/03/2020 are shown in Fig. 1, and cranial diffusion MRI images of the same date are shown in Fig 2.

It was decided that the patient should be followed

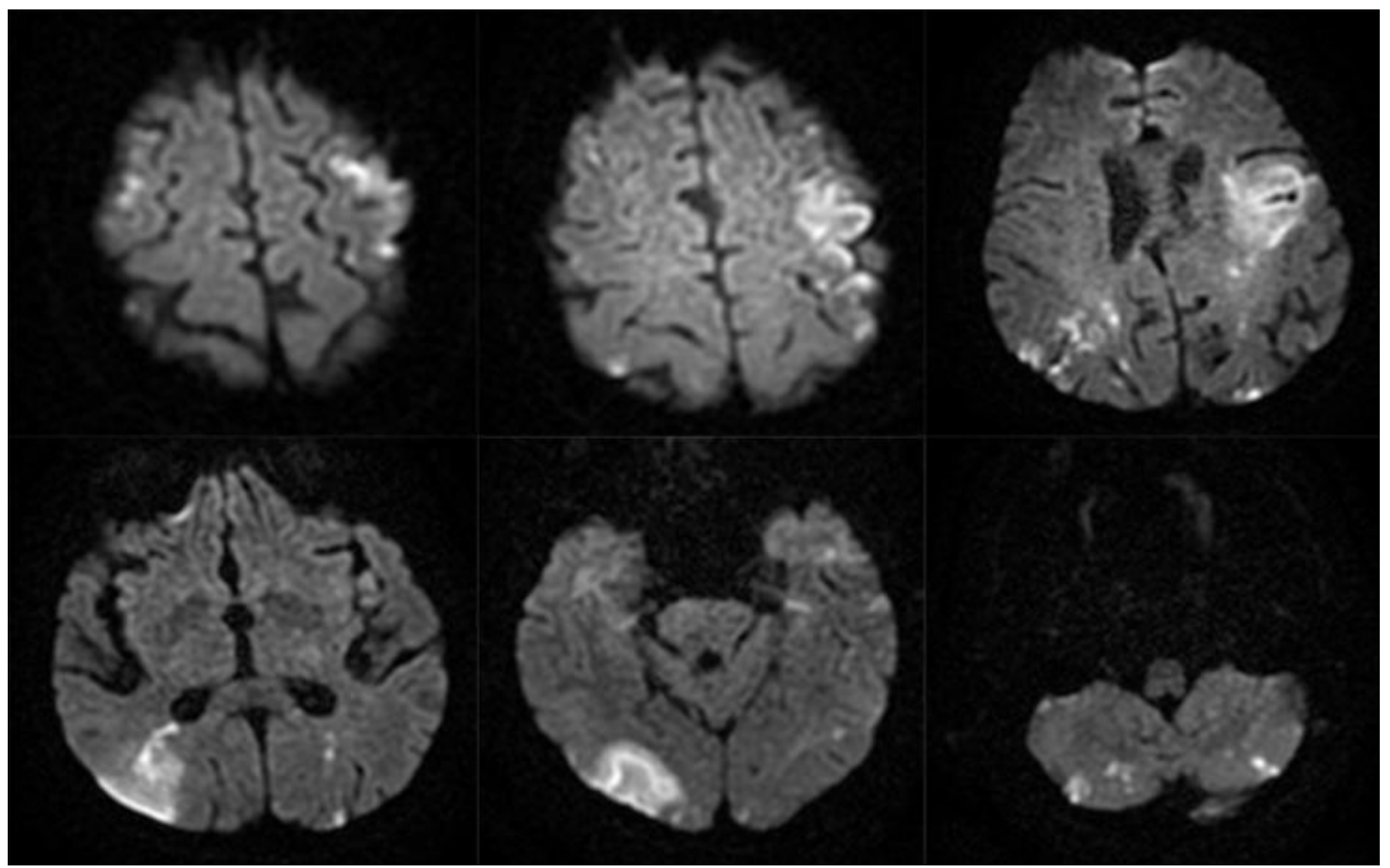

Fig. 2. Cranial diffusion magnetic resonance imaging 


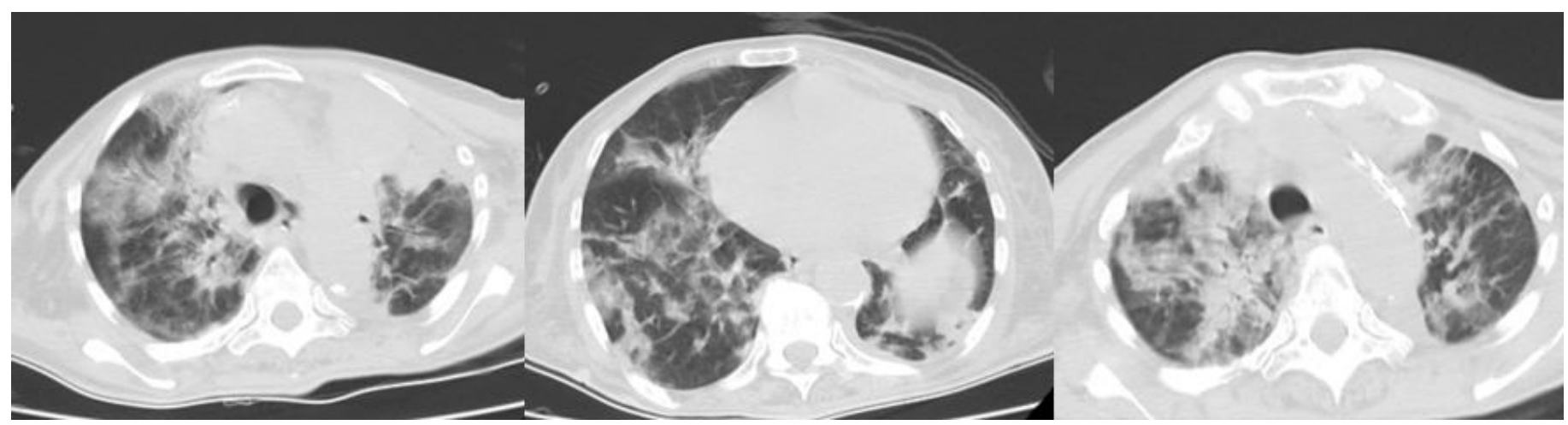

Fig. 3. Thorax computed tomographic imaging

up in intensive care unit since she had extensive cranial infarctions and due to her general condition. PCR sample of the patient was sent on 29/03/2020, after the patient's husband was taken under treatment in the hospital due to his positive COVID-19 PCR test and her thorax tomography was repeated. Significant changes were observed in the performed thorax tomography compared to the previous one. Images of thorax tomography of 29/03/2020 are shown in Fig. 3. Examinations in intensive care unit on 29/03/2020 showed Ferritin as $>2000 \mu \mathrm{g} / \mathrm{L}$, D-dimer as $816 \mu \mathrm{g} / \mathrm{L}$ FEU, fibrinogen as $493 \mathrm{mg} / \mathrm{dL}$, platelet as $107 \mathrm{~K} / \mu \mathrm{L}$, hemoglobin as $11 \mathrm{~g} / \mathrm{dL}$ and lymphocyte as 0.253 $\mathrm{K} / \mathrm{uL}$. No significant change was observed in lactate, $\mathrm{CO}_{2}$ or $\mathrm{pH}$. The patient who had positive PCR test passed away in intensive care unit on 31.03.2020.

\section{DISCUSSION}

In patients presenting to the emergency department with stroke findings may have COVID-19 disease. It was reported that COVID-19 disease was detected in one patient presenting with only weakness of the right extremity [1]. The reason for this may be microtrombus caused by COVID-19 disease. Especially, severe COVID-19 patients may face the risk of both thrombosis and hemorrhage [2]. COVID-19 disease was severe in $60 \%$ of patients with high D-dimer level [3]. When patients who died from COVID-19 and who survived were compared, high level of Ddimer supports this [4]. Our patient's D-dimer level was also high and supports these results.

Ferritin level also started to emerge as an important finding in COVID-19 patients. It can be observed that ferritin level rises to the values expressed in thousands [5]. High ferritin levels in COVID-19 patients are associated with death [6]. Our patient's ferritin being $>2000 \mu \mathrm{g} / \mathrm{L}$ corresponds to this result.

The presence of lymphopenia in patients is considered as a severe disease factor for COVID-19 [7]. Although our patient did not have lymphopenia, on 23/03/2020, when she first presented to the emergency service, she developed lymphopenia during her hospital stay. During the six-day period, lymphocyte count of our patient decreased from $1.81 \mathrm{~K} / \mathrm{uL}$ to $0.25 \mathrm{~K} / \mathrm{uL}$.

There are publications stating that the COVID-19 patients with critical conditions have increased fibrinogen levels $[4,8]$. Fibrinogen count of our patient was $493 \mathrm{mg} / \mathrm{dL}$ and was higher than normal.

Hypercoagulability is one of the tables that is encountered in cancer patients [9]. The fact that our patient had a history of lung cancer and also was diagnosed with COVID-19 may strengthen the possibility of hypercoagulability and cause thrombosis-related stroke. Our patient was also a diabetes patient. Diabetes disease has been reported to adversely affect the prognosis of COVID-19 [10]. It is also frequently reported that higher mortality is observed in elderly COVID-19 patients with hypertension [11]. Our patient had hypertension and therefore being under treatment may also be a factor in poor prognosis course. This case may give us an idea that when the number of comorbid conditions increase, mortality ratio may increase.

\section{CONCLUSION}

COVID-19 disease progresses with symptoms 
especially such as cough, fever and shortness of breath. Most patients overcome the disease with mild symptoms. However, it should be taken into consideration that COVID-19 disease may cause a more severe clinical table and may be encountered as a stroke case, except for symptoms such as cough and fever. In places where COVID-19 disease is observed commonly, it would be appropriate for emergency physicians to take COVID-19 disease into consideration in stroke cases.

\section{Informed consent}

Written informed consent was obtained from the patient's family for publication of this case and any accompanying images.

\section{Conflict of interest}

The authors declared that there are no potential conflicts of interest with respect to the research, authorship, and/or publication of this article.

\section{REFERENCES}

1. Zhai P, Ding Y, Li Y. The impact of COVID-19 on ischemic stroke: a case report. 2020 Mar 31 [cited 2020 Apr 12]; Available from: https://www.researchsquare.com/article/rs-20393/v1.

2. Xu JF, Wang L, Zhao L, Li F, Liu J, Zhang L, et al. Risk assessment of venous thromboembolism and bleeding in
COVID-19 patients. 2020 Mar 24 [cited 2020 Apr 12]; Available from: https://www.researchsquare.com/article/rs-18340/v1.

3. Thachil J, Tang N, Gando S, Falanga A, Cattaneo M, Levi M, et al. ISTH interim guidance on recognition and management of coagulopathy in COVID-19. J Thromb Haemost 2020;18:10236.

4. Tang N, Li D, Wang X, Sun Z. Abnormal coagulation parameters are associated with poor prognosis in patients with novel coronavirus pneumonia. J Thromb Haemost 2020;18:844-7.

5. Shoenfeld Y. Corona (COVID-19) time musings: our involvement in COVID-19 pathogenesis, diagnosis, treatment and vaccine planning. Autoimmun Rev 2020;19:102538.

6. Zhou F, Yu T, Du R, Fan G, Liu Y, Liu Z, et al. Clinical course and risk factors for mortality of adult inpatients with COVID-19 in Wuhan, China: a retrospective cohort study. Lancet 2020;395:1054-62.

7. Bermejo-Martin JF, Almansa R, Menéndez R, Mendez R, Kelvin DJ, Torres A. Lymphopenic community acquired pneumonia as signature of severe COVID-19 infection. J Infect 2020;80:e23-4.

8. Zhang Y, Cao W, Xiao M, Li YJ, Yang Y, Zhao J, et al. [Clinical and coagulation characteristics of 7 patients with critical COVID-2019 pneumonia and acro-ischemia]. Zhonghua Xue Ye Xue Za Zhi 2020;41:302-7. [Article in Chinese]

9. Davies NA, Harrison NK, Sabra A, Lawrence MJ, Noble S, Davidson SJ, et al. Application of ROTEM to assess hypercoagulability in patients with lung cancer. Thromb Res 2015; 135:1075-80.

10. Guo W, Li M, Dong Y, Zhou H, Zhang Z, Tian C, et al. Diabetes is a risk factor for the progression and prognosis of COVID-19. Diabetes Metab Res Rev 2020:e3319.

11. Shahid Z, Kalayanamitra R, McClafferty B, Kepko D, Ramgobin D, Patel R, et al. COVID-19 and older adults: what we know. J Am Geriatr Soc 2020;68:926-9. 